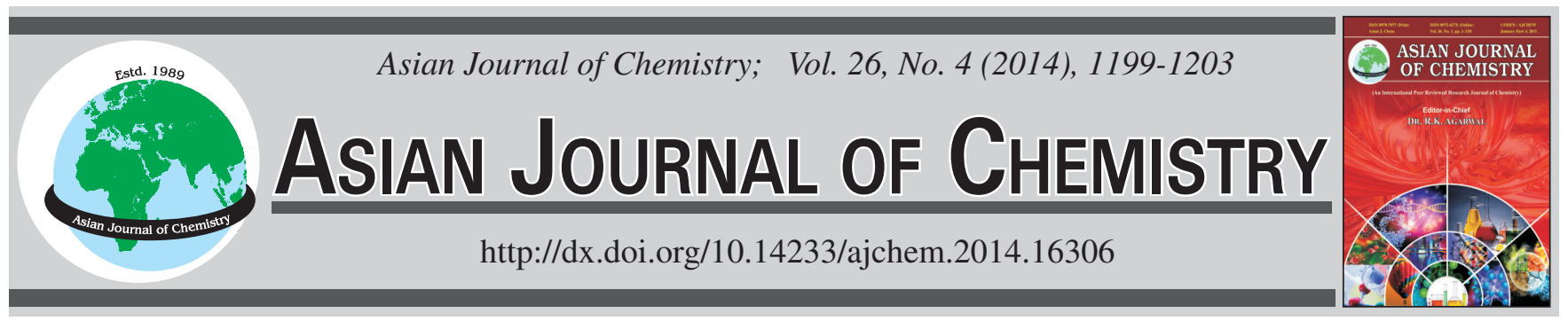

\title{
Study on Polymer Formation and Film-forming Properties Under Catalysis of Transglutaminase Between Casein and Keratin
}

\author{
XIAO-Zhou Su and Hong-Ru WAng*
}

College of Resources and Environment, Shaanxi University of Science and Technology, Xi'an 710021, P.R. China

*Corresponding author: Tel/Fax:+86 29 86132565; E-mail: feitianshenzhou@163.com

\begin{abstract}
Different mass ratio of casein and keratin were used to produce heteropolymer under the treatment of transglutaminase. The molecular weight of the polymer was investigated by SDS-PAGE. Films mechanical and water vapor barrier properties of the blended film were also studied. Film morphology was observed by using scanning electron microscopy. The crosslinking between casein and keratin proved by electrophoresis and the molecular weight of the new polymer was presumed about $50-70 \mathrm{kDa}$. The casein/keratin blended films showed an increase in elongation and water vapor permeability with transglutaminase treatment. The synergistic effect can be seen from the elongation value of the transglutamiase treatment casein/keratin blended filmed at the mass ratio of $4: 1$. The value of the tensile strength of the blended films decreased significantly with the increasing content of keratin.
\end{abstract}

Keywords: Casein, Keratin, Crosslinking, Heteropolymer, Film-forming property.

\section{INTRODUCTION}

Microbial transglutaminase is a kind of enzyme that can catalyze acyl-transfer reactions between $\lambda$-carboxyamide groups of glutamine residues and $\varepsilon$-amino of lysine residues. It catalyzes the crosslinking reaction between the same and different proteins, resulting in the formation of homopolymer and heteropolymers. The effect of transglutaminase treatment on polymer properties had been studied for many protein: casein $^{1}$, gelatin ${ }^{2}$, soybean protein ${ }^{3-4}$, whey $\operatorname{protein}^{5}$, keratin ${ }^{6}$, myofibrillar protein ${ }^{7}$, glutelins ${ }^{8-11}$. In most of these researches, transglutaminase was used in the crosslinking of one single type of protein. However, the crosslinking reaction can also take place between the glutamines and lysines of different types protein, for example, whey protein and 11 s globulin ${ }^{12-13}$, pepsinsolubilized elastin-collagen ${ }^{14}$, myofibrillar protein and soy protein $^{15}$, whey protein and zein hydrolysate ${ }^{16}$, wheat protein and lupine protein, gelatin and soy protein ${ }^{17}$, gelatin and whey $\operatorname{protein}^{18}$.

There were few reports on the crosslinking reaction between casein and other proteins by using transglutaminase. Kurth $^{19}$ found that under the conditions of $\mathrm{pH}=5.5-7.0, \mathrm{~T}=$ $45^{\circ} \mathrm{C}$, reaction time $=16 \mathrm{~h}$, mass ratio $=1: 2.5$, casein and myosin cross-linked and produced polymer with high molecular weight by using transglutaminase. Oh et al. ${ }^{16}$ used transglutaminase to make films from casein and zein hydrolyzate. Tensile strength of cross-linked films increase by $41 \%$, water vapor permeability was improved obviously. Chambi and Grosso $^{20}$ used transglutaminase to produce a gelatin/casein cross-linked polymer which molecular weight was about 40$200 \mathrm{KDa}$. The films contain of these two proteins had a synergic increasement in elongation compared with films made of proteins isolated.

At present, only Cui et al. ${ }^{21,22}$ used casein and gelatin to crosslink to the surface of the wool by using transglutaminase, leading to the improvement of the wool scalability and tensile strength. Before reaction, wool was treated with potassium permanganate solution. The cysteine was oxidated and the hydrophily of the wool surface was improved. This treatment enhanced the surface affinity and reactivity between casein/ keratin and gelatin/keratin, resulting in the amide group transferred reaction between casein/keratin and gelatin/ keratin. But this research did not involve in the reaction happened in the solution absolutely.

Casein and keratin are heterologous proteins with the isoelectric points of 4.6 and 4.8 , respectively. They had better miscibility in solutions in our preliminary experiment. Studies revealed that casein exhibits complicated aggregates micelles with spherical and linear features in solution. It presents a loosened conformation and has no typical secondary and tertiary structure. It tends to undergo structural alterations in the presence of external factors. Casein has many amide group and keratin has amount of amino group by alkaline treatment. All these conditions implied casein and keratin has a great 
possibility to crosslink by transglutaminase in the aquatic media. So we can use these two different proteins to produce heteropolymer in solution. This novel polymer has special material properties and biological compatibility, it can be developed into ecological coatings and biological membrane.

\section{EXPERIMENTAL}

Casein $($ particle size $=30$ mesh, protein $=95 \%$, fat $=$ $2 \%$, lactose $=0.2 \%$, ash $=2.5 \%$, moisture content $=12 \%$, free acidity $=0.15 \mathrm{~mL} / \mathrm{g}$ ) was supplied by Fonterra Co-operative (Group Auckland, New Zealand). The wool was collected after degreasing and washing and then dissolved in the solution by adding sodium sulphide. The keratin solution was left at room temperature until it completely dissolved. Diluted hydrochloric acid was added in the supernatant and the keratin sediment was purified and dried. Streptoverticillium $\mathrm{Ca}^{2+}$ independent transglutaminase was supplied by Jiang Su Yiming bio-technology company (TG-B, activity:100 u/g China). Glycerol was used as the plasticizer for the film formation. The entire reagents were analytical grade.

Film formation and conditioning: Casein and keratin solution with concentration of $7 \%$ were prepared. The casein was dissolved in distilled water with $\mathrm{NaOH}$ was added gradually until pH 7.5 was reached. Magnetic stirring was needed in the course of dissolution. Keratin powder was put into distilled water and $\mathrm{NaOH}$ was added until it completely dissolved at the concentration of $7 \%$. Different mass of protein solutions were mixed according to the desired proportion. The $\mathrm{pH}$ of mixed solution was adjusted to 7.5 and then put into the water bath at temperature of $50{ }^{\circ} \mathrm{C}$ for $30 \mathrm{~min}$ to disperse the protein. Glycerol $(30 \mathrm{~g} / 100 \mathrm{~g}$ of dry protein) was incorporated and the enzyme $(15 \mathrm{U} / \mathrm{g})$ was added. The solution was incubated for $1 \mathrm{~h}$ at temperature of $50{ }^{\circ} \mathrm{C}$, then the enzyme was inactivated at $80^{\circ} \mathrm{C}$ for $10 \mathrm{~min}$ in water bath. Polyfluortetraethylene plates with groove was used to cast the films. The films dried at room temperature for $48 \mathrm{~h}$ and then put into the desiccators [saturated solution of $\mathrm{Mg}\left(\mathrm{NO}_{3}\right)_{2} \cdot 6 \mathrm{H}_{2} \mathrm{O}$ ] for $48 \mathrm{~h}$ before testing.

Measurement of crosslinking: SDS-polyacrylamide gel electrophoresis (SDS-PAGE) was used to study the crosslinking of the protein. The gels include a $4 \%(\mathrm{w} / \mathrm{v})$ acrylamide stacking gel and a $10 \%(\mathrm{w} / \mathrm{v})$ acrylamide running gel, which was $0.5 \mathrm{~mm}$ thick. $20 \mathrm{mg}$ of the cross-linked solution was dissolved in $2 \mathrm{~mL}$ of sample buffer [tris- $\mathrm{HCl} \mathrm{pH} 6.80 .0625$ $\mathrm{mol} / \mathrm{L}$, containing $2 \%(\mathrm{w} / \mathrm{v}) \mathrm{SDS}, 12 \%(\mathrm{v} / \mathrm{v})$ glycerol, $5 \%$ mercaptoethanol and $0.1 \%$ (w/v) bromophenol blue] and heated at $95{ }^{\circ} \mathrm{C}$ for $3 \mathrm{~min} .5 \mu \mathrm{L}$ sample were loaded into each lane of gel. Electrophoresis was proceeded at $100 \mathrm{~mA}$ until the tracker dye reached the bottom of gels. The gels were then stained with $0.25 \%$ (w/v) coomassie brilliant blue R-250 [in $10 \%(\mathrm{v} / \mathrm{v})$ acetic acid: $45 \%(\mathrm{v} / \mathrm{v})$ methanol] and destained with $10 \%(\mathrm{v} / \mathrm{v})$ acetic acid containing $25 \%(\mathrm{v} / \mathrm{v})$ methanol. The protein molecular markers for calibration were 6.5-200 kDa (Cata No CB800002 Protein Marker II, California Bioscience, USA.

Mechanical properties: Mechanical properties test carried out according to the ASTM D882-98 (ASTM, 1998) and included tensile strength and elongation measurements. The universal material testing machine (TCS-2000, GOTECH incorporated company Taiwan) was used for testing. The films were clipped into a dumbbell-like shape and $8 \mathrm{~cm}$ length and $1.5 \mathrm{~cm}$ width. Film thickness was acquired by calculating the mean of five measurements using the digital micrometer. All the measurements were done in triplicate.

Water vapor permeability (WVP): According to the ASTM E96-95 (ASTM, 1995) method, the water vapor permeability of the blended films was measured. The films were fixed by microcrystalline paraffin and put onto the aluminum cells containing anhydrous calcium chloride, then placed in desiccators containing saturated $\mathrm{NaCl}$ solutions. The cells were weighted daily for a week to determine the mass variation. The water vapor permeability was measured in triplicate.

Scanning electron microscopy: The films were taken out from the desiccator which containing silica gel for 10 days and liquid nitrogen was dropped on the films to fracture them into small pieces. The cross-section of the films was observed after coated with gold. Hitachi S-4800 field emission scanning electron microscope was used to examine the films at $1 \mathrm{kV}$. The magnification was set $5 \mathrm{~K}$ and $120 \mathrm{~K}$.

Statistical analysis: Statistical 8 was used to analyze the variance of the data. When the ANOVA test indicated a significant difference $(\mathrm{p}<0.05)$ among the means, then Tukey's test was used to determine which means differed significantly.

\section{RESULTS AND DISCUSSION}

The casein was immersed in small amount of water and the $\mathrm{NaOH}$ solution was added gradually until it completely soaked through at the $\mathrm{pH}$ of 7.5 by 2 days. Distilled water was added under constant magnetic stirring until the solution became transparent. The keratin solution used for film taken from the supematant. The $\mathrm{pH}$ of the mixed solution was carefully adjusted to 7.5 and avoided of adjusting back and forth due to the influence of the ion strength.

Analyses of the SDS-PAGE (Fig. 1): Lane 1 was the results of electrophoresis of the casein with transglutaminase treatment and lane 2 was the casein without transglutaminase treatment. The pattern similar to the results made by Chambi and $\mathrm{Grosso}^{20}$. Lane 3 and lane 4 were the keratin with transglutaminase treatment and without transglutaminase treatment. There was no obviously band in lane 3 and 4 because of the low molecular of the keratin dissolved by alkali and the molecular weight about 6-9 kDa by severe alkali hydrolysis reported by Cardamone ${ }^{23}$. The molecular weight for the keratin dissolved by alkali in this experiment was $8000 \mathrm{Da}$ by gel filtration chromatography method. Lane 5-9 match to the casein/keratin $=4: 1,2: 1,1: 1,1: 2,1: 4$, in the presence of transglutaminase. We can see the $\alpha s_{1}, \alpha s_{2}, \beta, \kappa$ components of the casein between $20 \mathrm{KDa}$ nearby in lane $\mathrm{e}^{5-9}$. Besides, the bands between 43-68 KDa can also be observed but not clearly than the casein components. The bands existed in 43-68 KDa were the proofs of the polymer formed between casein and keratin by transglutamiase treatment. Among them, the band of casein/ keratin $=2: 1$ was most clearly. The reason of polymer bands were not clearly then casein components was that there were part of casein and keratin participated in the crosslinking reaction by transglutaminase due to the limitation of the crosslinking reaction. This can be assumed that different 


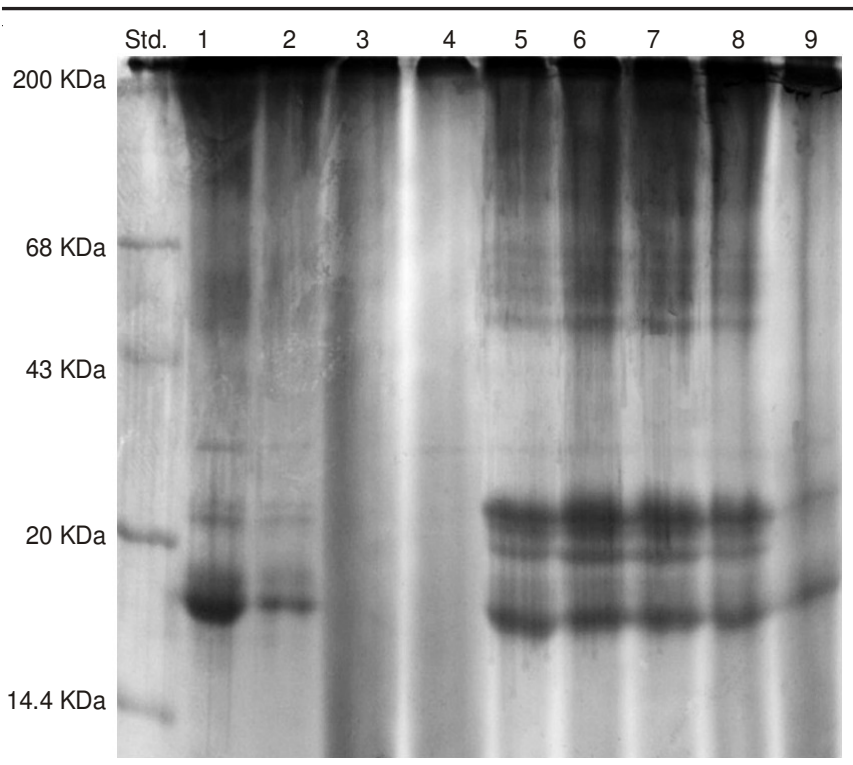

Fig. 1. SDS-PAGE electrophoresis of the cross-linked heteropolymer at different mass ratio of casein and keratin: lane1 (casein, with transglutaminase), lane2 (casein, without transglutaminase), lane3 (kerain, with transglutaminase), lane4 (keratin, without transglutaminase), lane5 (casein $/$ keratin $=4: 1$, with transglutaminase), lane6 (casein/keratin $=2: 1$, with transglutaminase), lane7 $($ casein $/$ keratin $=1: 1$, with transglutaminase), lane 8 (casein/keratin $=1: 2$, with transglutaminase), lane 9 (casein/keratin $=1: 4$, with transglutaminase)

components of casein were joint together by 1 or 2 keratin molecules and heteropolymer formed between casein and keratin.

Tensile strength: The tensile strength of casein films varied from 1-50 MPa according to the previous studies. Chen et $a l .{ }^{24}$ reported that the value of the casein films was $48 \mathrm{MPa}$. Chen et al. ${ }^{25}$ reported that the value was 1.6 MPa. Reichl et al. ${ }^{26}$ observed that the value of the keratin films prepared by reduction method was $16 \mathrm{MPa}$. Tanabe et al. ${ }^{27}$ found that the tensile strength of the films mixed by different proportions of the keratin and chitosan changed from 0 to $44 \mathrm{MPa}$. The great fluctuation of the values of the protein films primary due to the different measuring method and samples' moisture, shape, test temperature and other factors can affect the measurement.

The tensile strength of the blended films in all the proportions cross-linked with or without transglutaminase decreased greatly compared with the casein film solely and varied as a function of the proportions of the 2 proteins ( $p>0.05$ ) (Fig. 2). So the introduction of the keratin into the casein films resulted the decline of the tensile strength. $7 \%$ of the keratin solution dissolved by alkali can not to form film because of the low molecular weight. Similarly, Oh et al. ${ }^{16}$ found that the addition of zein hydrolyzate into the whey protein resulted in the decrease by $35-41 \%$ of the blended films. Chambi and Grosso $^{20}$ reported that the film formed by casein and gelatin also had the similar results. Casein is generally regarded as non-ordinate proteins, it contains small amount of $\alpha$-helical or $\beta$-sheet structure conformations and has unorganized matrix. $63 \%$ of the structures exhibited random or twisted conformations. So when another protein with low molecular weight introduced, it may reinforce the disordered matrix structure and leading to low tensile strength.

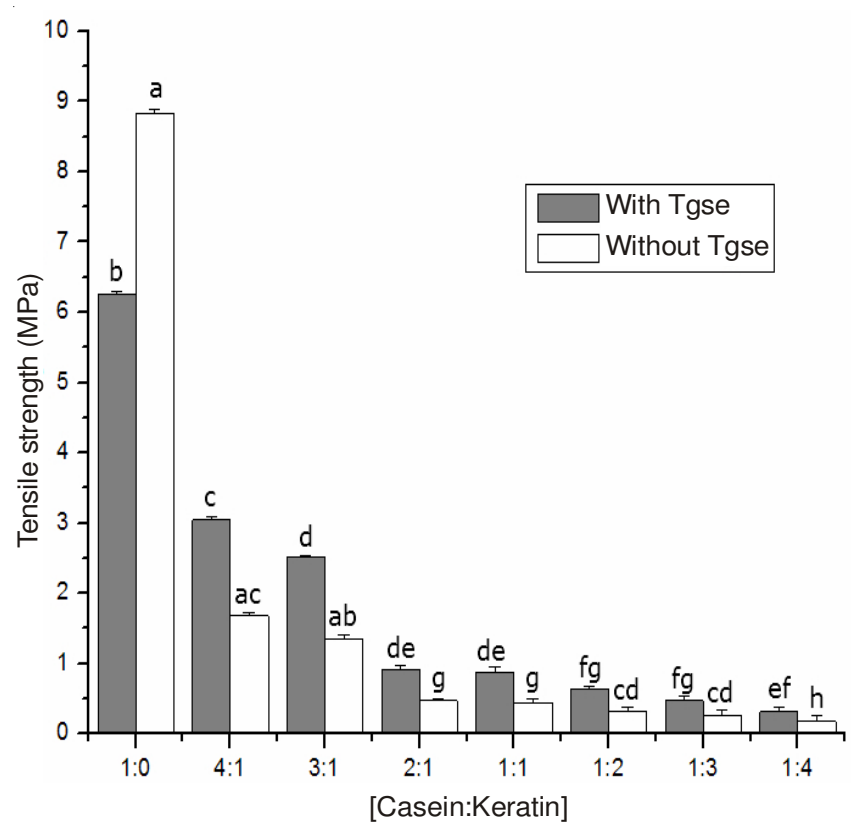

Fig. 2. Tensile strength of the casein/keratin blended films with or without transglutaminase. Note: different letters represent significant different $(\mathrm{p}<0.05)$ between the means in tukey test

As for the enzyme treatment, the tensile strength of the films also exhibited significant changes ( $p>0.05$ ) (Fig. 2) compared to the films without transglutamiase. Similarly, Yildirim and Hettiarachchy ${ }^{12}$ observed that the tensile strength of blended films contained $11 \mathrm{~S}$ globulin and whey, soy and pectin cross-linked with transglutaminase was twice the value of films without transglutaminase. These may be due to the formation of more stable network because of the crosslinking effect.

Elongation: The films contained two proteins presented greater elongation than casein films individually with or without transglutamiase. The values of the elongation for films crosslinked with transglutaminase generally higher than the films without transglutaminase and the elongation of the films under the casein/keratin proportion of $4: 1$ increased by $38 \%$ compared to the films without transglutaminase (Fig. 3) ${ }^{16}$. Oh et al. ${ }^{16}$ also found that the elongation of the pepsin-solubilized elastin-collagen film crosslinking by transglutamiase more than 1.4 times of the film without transglutaminase ${ }^{14}$. The interaction between different proteins may lead to phase separation, precipitation or synergistic effect according to the Howell ${ }^{28}$. In this study, the synergistic effect displayed in the reinforcement the elongation of the films composed of different proportions of casein and keratin. The polymer chains in the blended films interacted by van der waals forces and linked at some covalent bonding sites. These sites limit the relative movement of the chains. The crosslinking by transglutamiase can increase the elongation because new bonding sites formed by the amide bonds and the mobility of the chains increase. It reported that the introduction of plasticizers into films can improve the elongation values because it decreased the molecular attractive forces and enhanced the mobility of polymer chains. So when hydrophobic keratin added into the casein, the similar effect resulted in increase of elongation values in the absence of transglutaminase. 


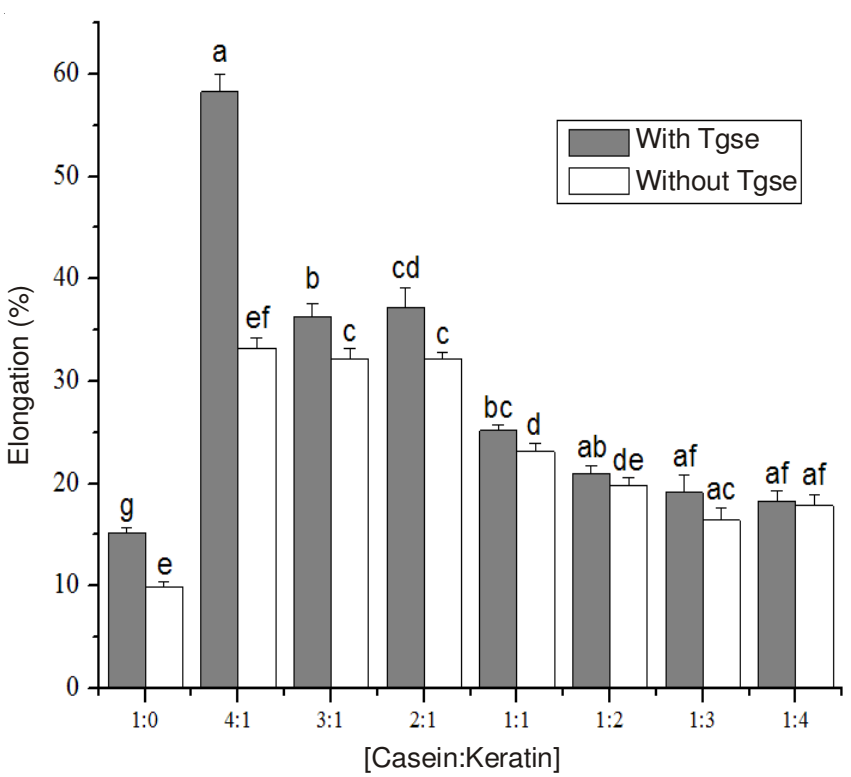

Fig. 3. Elongation of the casein/keratin blended films with or without transglutaminase. Note: different letters represent significant different $(\mathrm{p}<0.05)$ between the means in tukey test

Water vapor permeability: The water vapor permeability of the cross-linked films by transglutaminase presented significant differences compared with the same blended films without enzymatic treatment (Fig. 4). The phenomena also took place in elongation test for films. The increase in polymer chain mobility also resulted in the increase in the water diffusion coefficient and enhanced the water vapor permeability. Similar result also obtained by Chambi and Grosso ${ }^{20}$ for casein and gelatin blended films treated with transglutaminase. Further-

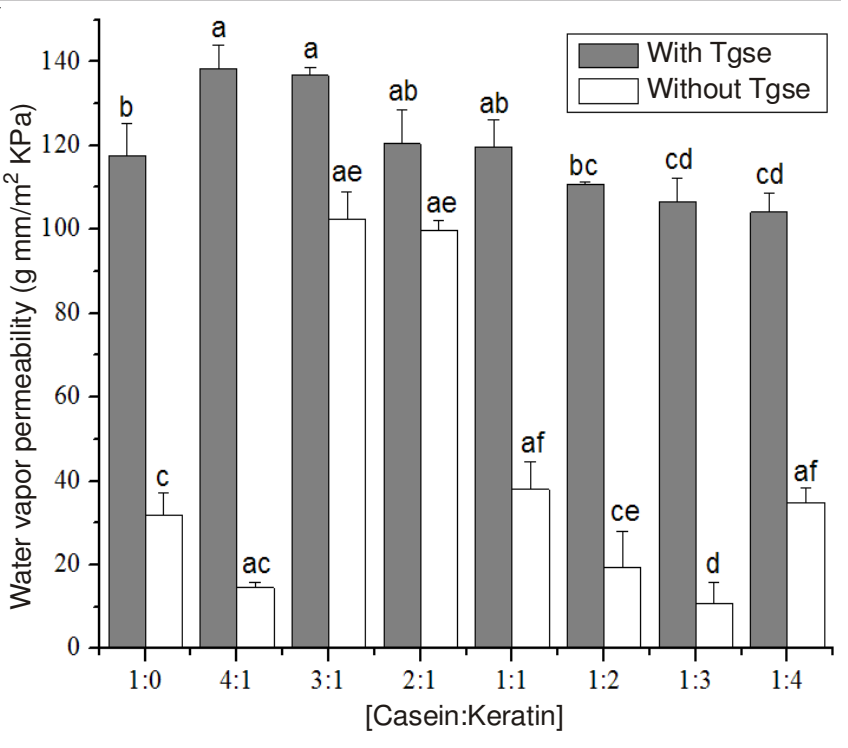

Fig. 4. Water vapor permeability of the casein/keratin blended films with or without transglutaminase. Note: different letters represent significant different $(\mathrm{p}<0.05)$ between the means in Tukey test

more, the water vapor permeability of the enzymatic treated whey protein, soy $11 \mathrm{~S}$ globulin and the two protein mixture enhanced reported by Yildirim and Hettiarachchy ${ }^{12}$. The crosslinking effect caused by amide bond changed the structure of the polymer matrix and may increase the number of the hydrophobic amino acids existed on the surface of films. Keratin usually classified into the protein with strong hydrophobicity. So when keratin was added into the casein, the films hydrophobicity changed dramatically without the enzymatic treatment.

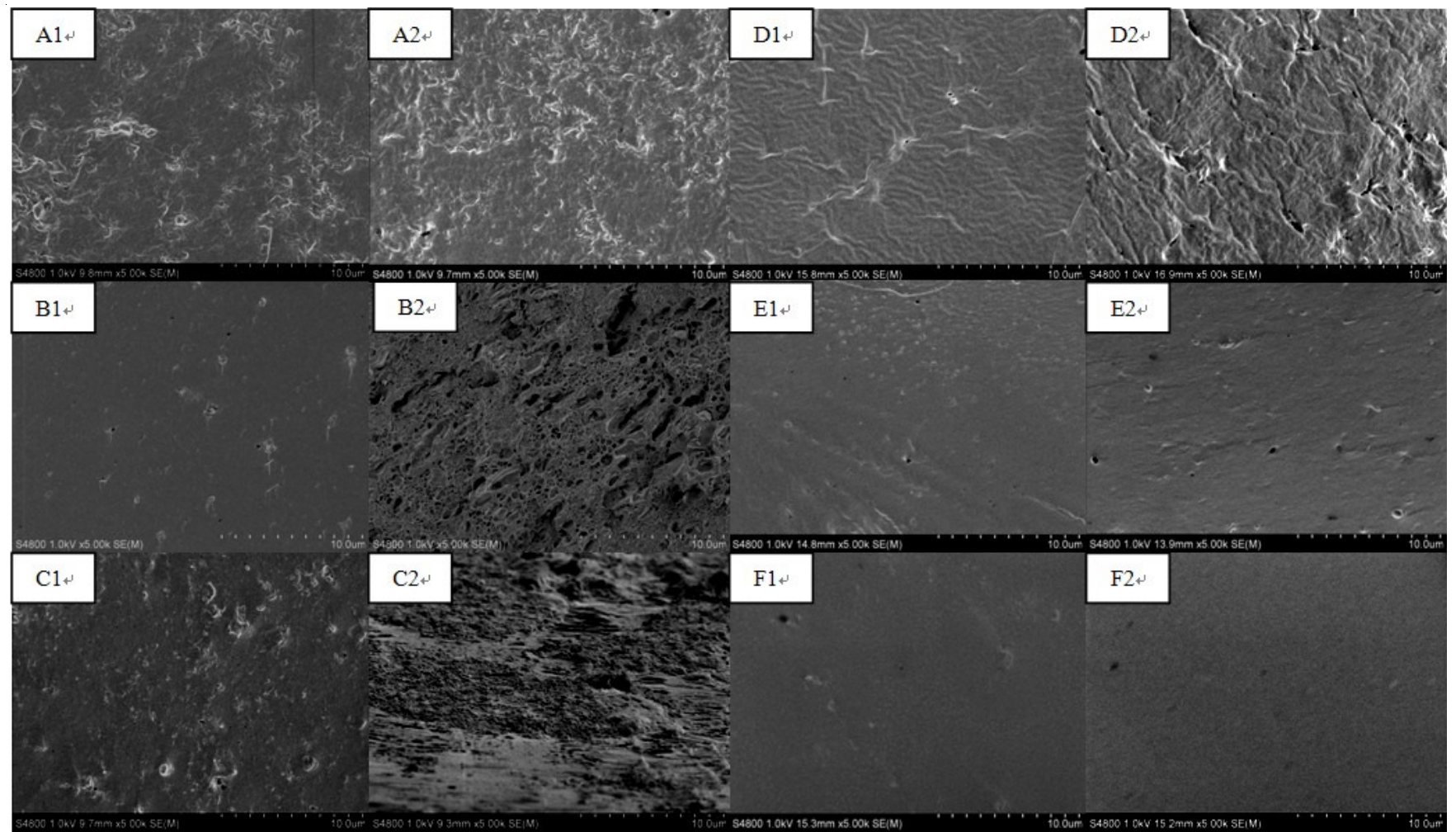

Fig. 5. SEM images of the transversal section of casein/keratin blended films with or without transglutaminase. A1-F1 represented casein/keratin blended films with transglutaminase at the mass ratio of 1:0, 4:1, 2:1, 1;1, 1:2, 1:4. A2-F2 represented casein/keratin blended films without transglutaminase at the mass ratio of 1:0, 4:1,2:1, 1;1, 1:2, 1:4. Magnification: $5000 \times$ 
Film morphology: The images of the film's transversal section observed by scanning electron microscopy were showed in Fig. 5. A1-F1 represented casein/keratin blended films with transglutaminase at the mass ratio of 1:0, 4:1, 2:1, $1 ; 1,1: 2,1: 4$. A2-F2 represented casein/keratin blended films without transglutaminase at the mass ratio of 1:0, 4:1, 2:1, $1 ; 1,1: 2,1: 4$. Fig. A2-F2 presented an uneven surface of the films which without transglutamiase treatment. Fig. A1-F1 presented a relative flat surface which treated with transglutamiase compared with Fig. A2-F2. This demonstrated that the structure of the blended films treated with transglutaminase became more compact due to the crosslinking effect. The covalent amido bond newly formed stabilized the protein network and also resulted the alteration in elongation, tensile strength and water vapor permeability.

The casein/keratin blended films without transglutaminase treatment at the mass ratio of 4:1(B2), 2:1(C2) and 1:1(D2) exhibited rough surface with depressions and ridges and the film at the mass ratio of 4:1(B2) also exhibited a spongy structure. This may be due to the interaction of the molecular chains between the heterologous proteins and casein/keratin blended micelles exhibited specially structure and conformations. This structure may resulted the improvement of the elongation for the transglutaminase untreated films. Casein/ keratin blended films at the mass ratio of 4:1(B1, B2) were further detected at the magnification of 120000 (Fig. 6). The figure showed that film treated with transglutaminase composed of compact particles which diameters under 100 $\mathrm{nm}$ mostly and the film treated without transglutaminase composed of particles greater than $100 \mathrm{~nm}$ mostly. The significant growth in the elongation with transglutaminase may be due to the effect of the nano-paticles.

Fig. 5. A1, A2 showed that the casein film exhibited a little fibrous morphology without transglutaminase and this feature became weak for the films treated with transglutaminase or the introduction of keratin. This may result the tensile strength variation exhibited in Fig. 2 Casein film without transglutaminase treatment had the maximal tensile strength and value decreased when films treated with transglutaminase or adding of keratin. From B2-F2, the surface became flatten when more keratin was added and these more compact structures may result the decrease in tensile strength.

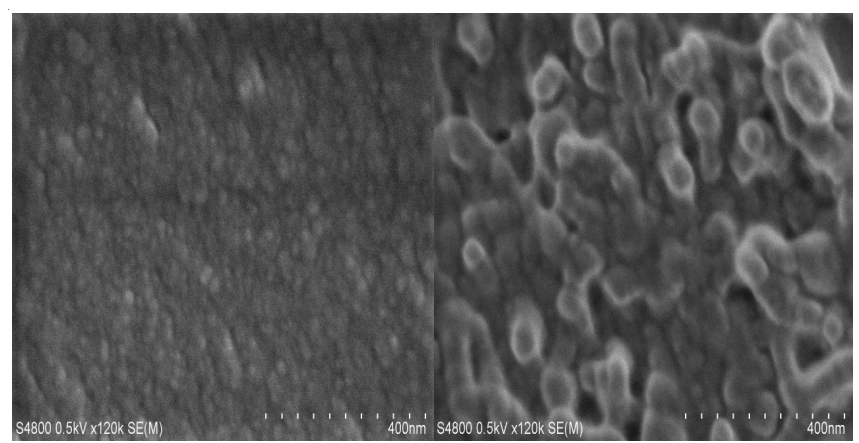

Fig. 6. SEM images of the transversal section of the casein/keratin blended films with or without transglutaminase at the mass ratio of $4: 1$. Magnification: $120000 \times$. Left figure: With transglutaminase treatment. Right figure: Without transglutaminase treatment

\section{Conclusion}

Casein and keratin was cross-linked by transglutaminase under certain conditions and heteropolymer formed with high molecular weight. Enzymatic treatment improved the elongation and water vapor permeability of the blended films significantly. The blended films of casein and keratin have an obviously synergistic effect on the film elongation at the mass ratio of 4:1 with transglutaminase treatment and the value of elongation was of $57.23 \%$. The introduction of the keratin into the casein films resulted a significant reduction in tensile strength. These modifications in film property have close relations with the micro-structure of the blended films.

\section{ACKNOWLEDGEMENTS}

The material in this work was supported by Jiangsu Yiming Bio-products Co.

\section{REFERENCES}

1. C. Partschefeld, U. Schwarzenbolz, S. Richter and T. Henle, Biotechnol. J., 2, 456 (2007).

2. M.M. Taylor, C. Liu, W.N. Marmer and E.M. Brown, J. Am. Leather Chemists Assoc., 98, 435 (2003).

3. N. Nio, M. Motoki and K. Takinami, Agric. Biol. Chem., 49, 2283 (1985).

4. E.E. Babiker, Food Chem., 70, 139 (2000).

5. L.T. Lim, Y. Mine and M.A. Tung, J. Agric. Food Chem., 46, 4022 (1998).

6. J.M. Cardamone and J.G. Phillips, Text. Res. J., 77, 277 (2007).

7. D.R. Kahn and I. Cohen, Biochim. Biophys. Acta, 668, 490 (1981).

8. M.C. Alexandre, Y. Popineau, G. Viroben, M. Chiarello, A. Lelion and J. Gueguen, Agric. Food Chem., 41, 2208 (1993).

9. C. Larré, S. Denery-Papini, Y. Popineau, G. Deshayes, C. Desserme and J. Lefebvre, Cereal Chem., 77, 121 (2000).

10. A. Basman, H. Köksel and P.K.W. Ng, J. Food Sci., 67, 2654 (2002).

11. A. Basman, H. Köksel and P.K.W. Ng, Eur. Food Res. Technol., 215, 419 (2002).

12. M. Yildirim and N.S. Hettiarachchy, J. Food Sci., 63, 248 (1998).

13. M. Yildirim and N.S. Hettiarachchy, J. Food Sci., 62, 270 (1997).

14. K. Takahashi, Y. Nakata, K. Someya and M. Hattori, Biosci. Biotechnol. Biochem., 63, 2144 (1999).

15. J.C. Ramirez-Suarez and Y.L. Xiong, Meat Science, 65, 899 (2003).

16. J.H. Oh, B. Wang, P.D. Field and H.A. Aglan, J. Food Sci. Technol., 39, 287 (2004).

17. A. Bonet, W. Blaszczak and C.M. Rosell, Cereal Chem., 83, 655 (2006).

18. E. Hernàndez-Balada, M.M. Taylor, J.G. Phillips, W.N. Marmer and E.M. Brown, Bioresour. Technol., 100, 3638 (2009).

19. L. Kurth, Food Technol. Austr., 35, 420 (1983).

20. H. Chambi and C. Grosso, Food Res. Int., 39, 458 (2006).

21. L. Cui, X. Fan, P. Wang, Q. Wang and G. Fu, Eng. Life Sci., 11, 201 (2011).

22. L. Cui, W. Qiang, W. Ping and H. Qingsong, J. Appl. Polymer, 4, 2053 (2011).

23. J.M. Cardamone, J. Mol. Struct., 969, 97 (2010).

24. H. Chen, R. Banerjee and E.J. Limoge, in eds.: I.A. Taub and R. Bell, Testing Mechanical Strengths of Edible Films; in Proceedings of Food Preservation 2000, Science and Technology Corporation, Hampton, VA, pp. 863-875 (1993).

25. H. Chen, J. Dairy Sci., 78, 2563 (1995).

26. S. Reichl, M. Borrelli and G. Geerling, Biomaterials, 32, 3375 (2011).

27. T. Tanabe, N. Okitsu, A. Tachibana and K. Yamauchi, Biomaterials, 23, 817 (2002).

28. N.K. Howell, Biopolymer Mixtures, University Press, Nottingham, pp. 329-347 (1995). 